\title{
3D Printed Bifunctional Triple-Band Heatsink Antenna for RF and Thermal Energy Harvesting
}

\author{
Azamat Bakytbekov, Zere Iman, Atif Shamim \\ Electrical Engineering Department \\ King Abdullah University of Science and Technology, KAUST \\ Thuwal, Saudi Arabia \\ Azamat.bakytbekov@kaust.edu.sa; zere.iman@kaust.edu.sa; atif.shamim@kaust.edu.sa
}

\begin{abstract}
Future IoT environment requires billions of wireless sensor nodes where energy consumption of these devices will be a concern. This problem can be solved by harvesting energy from ambient environment to power sensor nodes. Ambient RF and thermal energy have a great potential, as both of them are available in most of the places. When combining these two energy harvesters into one module, optimized design is required. This work presents a heatsink antenna, which serves as a part of a triple-band RF energy harvester (GSM900, GSM1800 and 3G) and as a heatsink which boosts the performance of the thermal energy harvester. The antenna has been designed by using Ansys HFSS and Solidworks in parallel for optimal RF and thermal performances.
\end{abstract}

Keywords-heatsink antenna, multiband antenna, multi-source energy harvester, RF energy harvester, thermal energy harvester

\section{INTRODUCTION}

Internet of Things (IoT) is a global infrastructure of smart physical objects based on different wireless sensors, which communicate with each other and make smart decisions in different scenarios. It is estimated that the number of smart things connected to the Internet will reach 75 billion in 2025 [1]. Therefore, huge number of IoT sensor nodes naturally implies the problem of energy consumption of these devices. Traditional way of recharging or replacing batteries might not be compatible with the new IoT reality. Ideally, IoT sensors must be selfpowered and low cost, considering the huge volume. One of the approaches to make the sensor nodes self-powered is to harvest energy from ambient environment and use it to power-up IoT sensor nodes. Considerable research has been conducted on different ambient energy harvesters such as radio frequency (RF) energy harvesters [2] that collect energy from standard cellular frequency bands such GSM and 3G; and thermal energy harvesters [3] that collect from diurnal temperature fluctuations of ambient environment. However, energy collected by these stand-alone ambient energy harvesters is quite low to run the wireless sensor nodes and continuous operation of sensors might not be achieved due to unpredictable availability of ambient energy sources. Thus, combining several ambient energy harvesters into one module can be an attractive solution as it 1) increases the energy collected from the ambient environment and, 2) ensures continuous operation of sensor nodes by compensating each other's absence. There are papers in the literature, which combine, particularly RF and thermal energy harvesters into one module [4, 5]. However, optimized integration of these harvesters has not been discussed in these

The authors acknowledge the KAUST Sensor Initiative, under award OSR-2015-Sensors-2700, for the financial support. papers. Ideal integration would be to have components of RF or thermal energy harvesters where they perform dual-function and boost the performance of both harvesters by achieving synergy between two. Heatsink antennas are ideal solution for this case as it 1) boosts the performance of the thermal harvester by augmenting the heat transfer through increased effective area, and 2) serves as an antenna for the RF energy harvester if the dimensions of the metallic fins are tuned to the required frequency bands [6].

This paper presents a bifunctional triple-band heatsink antenna, which is an important component of a multi-source ambient energy harvester based on RF and thermal energy harvesters. The component serves as an antenna of the RF energy harvester, which collects energy from GSM900, GSM1800 and 3G $(2.1 \mathrm{GHz})$ bands and is placed on four side faces of the thermal energy harvester box. Simultaneously, it works as a heatsink by increasing the heat transfer between ambient environment and inner filling of the box. Design of the heatsink antenna has been conducted on Ansys HFSS in terms of antenna performance and Solidworks in terms of thermal performance. Trade-off analysis of the RF and thermal performances is considered when optimizing the dimensions of the component.

\section{ANTENNA DESIGN}

The antenna of the RF energy harvester is triple-band and works at $900 \mathrm{MHz}, 1800 \mathrm{MHz}$ and $2100 \mathrm{MHz}$ frequencies at the same time. These GSM and 3G bands are selected based on the $\mathrm{RF}$ power measurements conducted prior to the antenna design and regarded as the most powerful bands compared to others such as $\mathrm{WiFi}$ [7]. In order to achieve multiband performance, fractal approach is utilized as it can provide several resonances based on recursively-generated geometry of fractals [8]. Cantor fractal (Fig. 1a) is used for the antenna design due to its simple structure. It consists of self-similar rectangular segments where main segment is copied, divided into three parts and the middle section is removed. The iteration is repeated several times to achieve the required bandwidth. In this case, two Cantor fractals are placed as two arms of a differential antenna. As it is shown in Fig. 1b, the Cantor fractal antenna is placed on two side faces of the thermal harvester box. In fact, the thermal harvester can accommodate two antennas on four side faces of the box and it is advantageous to have two RF energy harvesters because 1) it doubles the energy generated by RF energy harvester; 2) 360 degrees of azimuthal coverage is achieved; and 3) eventual goal 
(a)

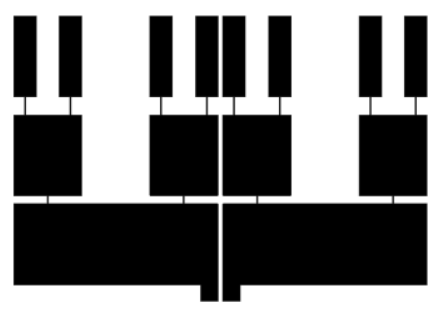

(b)

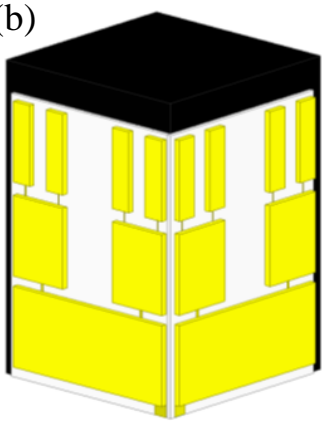

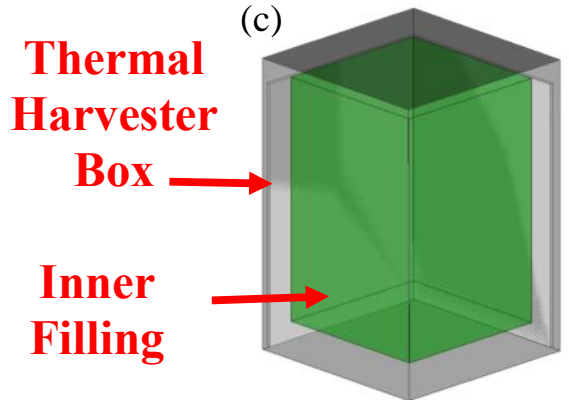

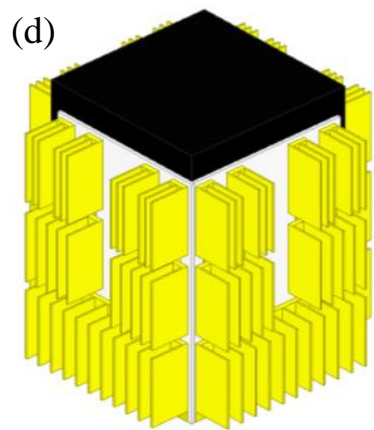

Fig. 1. (a) Cantor fractal (b) Fractal antenna on thermal harvester box (c) Body of the thermal harvester on HFSS (d) Heatsink antenna

of having heatsinks on all side faces of the box is realized. Prior to the antenna design process, a model of the thermal harvester has been built on HFSS as the body and inner filling of the thermal harvester (consists of some metal and dielectrics) affects the RF performance of the antenna (Fig. 1c). Therefore, dielectric constant and loss tangent of these materials have been characterized on impedance analyzer and inserted into HFSS model to achieve accurate results. The idea behind the Cantor fractal design of the triple-band antenna is that each iteration is responsible for one resonance. Thus, the first segment is responsible for the $2.1 \mathrm{GHz}$ frequency, as it has the shortest wavelength $(82.5 \mathrm{~mm})$. The length of the first segment is exactly equal to the wavelength at $2.1 \mathrm{GHz}$. Fins of the antenna have been installed on top of the first segment in a vertical direction (natural convection's direction). Through the analysis of the heatsink dimensions, it is found out that the parameters such as number of fins and thickness of fins do not affect the resonance of the antenna and thus their sizes are totally dictated by the best thermal performance. Thermal analysis has been done on Solidworks by tracking the best heat transfer between ambient environment and inner filling of the thermal harvester box. It is identified that the increase in number of fins and thickness of fins increases the heat transfer proportionally due to the more effective area. However, at some point it saturates and then degrades as the fins become too close to each other, which blocks the airflow through fins. The second and third resonances appear once we add two more iterations of rectangular segments with fins. Height of the fins is a parameter, which directly affects both RF and thermal performances of the heatsink antenna and therefore some trade-off analysis is conducted to achieve

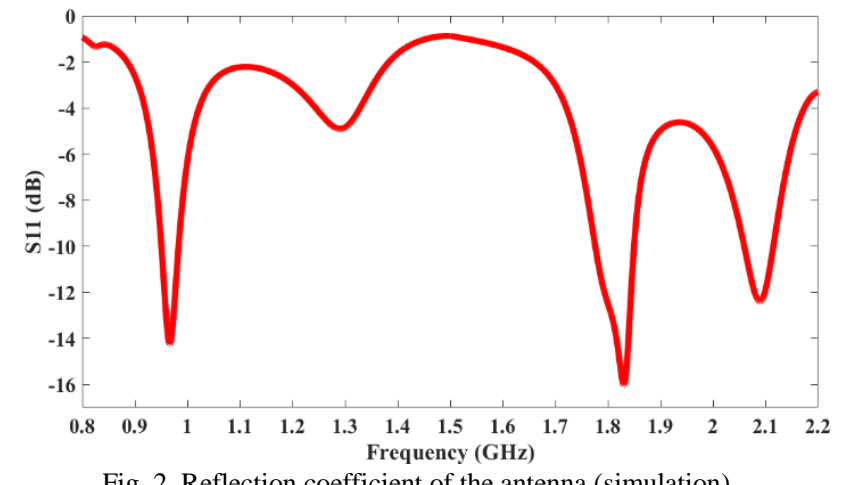

Fig. 2. Reflection coefficient of the antenna (simulation) compromise between antenna and heatsink functions. The final version of the antenna on HFSS and reflection coefficient of the antenna are presented in Fig. 1d and Fig. 2. Fabrication of the antenna will be done on metallic 3D printer and measurement results will be presented during presentation on the conference.

\section{CONCLUSION}

This paper presents a heatsink antenna for hybrid RF and thermal energy harvester. The antenna has been designed on two side faces of the thermal harvester box. The antenna operates at three frequency bands simultaneously $(900,1800,2100 \mathrm{MHz})$. The heatsink antenna has been designed by analyzing both RF and thermal performances in parallel through Ansys HFSS and Solidworks simulators respectively.

\section{REFERENCES}

[1] Statista Research Department, Internet of Things - number of connected devices worldwide 2015-2025, 2019, Accessed on 27 October 2019. [Online]. Available: https://www.statista.com/statistics/471264/iotnumber-of-connected-devices-worldwide/

[2] Azamat Bakytbekov, Thang Q. Nguyen, Cuong Huynh, Khaled N. Salama, Atif Shamim, Fully printed 3D cube-shaped multiband fractal rectenna for ambient RF energy harvesting, Nano Energy, Volume 53, 2018, Pages 587-595, ISSN 2211-2855.

[3] Anton L. Cottrill, Ge Zhang, Albert Tianxiang Liu, Azamat Bakytbekov, Kevin S. Silmore, Volodymyr B. Koman, Atif Shamim, Michael S. Strano, Persistent energy harvesting in the harsh desert environment using a thermal resonance device: Design, testing, and analysis, Applied Energy, Volume 235, 2019, Pages 1514-1523, ISSN 0306-2619.

[4] L. Guo, X. Gu, P. Chu, S. Hemour and K. Wu, "Collaboratively Harvesting Ambient Radiofrequency and Thermal Energy," in IEEE Transactions on Industrial Electronics.

[5] Jahariah Sampe, Nor Afidatul Asni Semsudin, Farah Fatin Zulkifli, Md. Shabiul Islam and Mohd. Zulhakimi Ab. Razak, 2017. Hybrid Energy Harvester Based on Radio Frequency, Thermal and Vibration Inputs for Biomedical Devices. Asian Journal of Scientific Research, 10: 79-87.

[6] L. Covert, J. Lin, D. Janning and T. Dalrymple, "Dual-Function 3-D Heatsink Antenna for High-Density 3-D Integration," 2007 IEEE International Workshop on Radio-Frequency Integration Technology, Rasa Sentosa Resort, 2007, pp. 26-29.

[7] A. Bakytbekov and A. Shamim, Additively Manufactured Triple-Band Fractal Antenna-on-Package for Ambient RF Energy Harvesting, 13th European Conference on Antennas and Propagation (EuCAP), Krakow, Poland, 2019, Pages 1-3.

[8] A. Bakytbekov, A. R. Maza, M. Nafe and A. Shamim, Fully inkjet printed wide band cantor fractal antenna for RF energy harvesting application, 11th European Conference on Antennas and Propagation (EUCAP), Paris, 2017, Pages 489-491. 